\title{
- ARTÍ́CULO ORIGINAL
}

\author{
Factores de riesgo asociados al pie diabético \\ Risk factors associated with diabetic foot \\ Autor: Ángel Daniel Enciso Rojas ${ }^{1}$ \\ Artículo recibido: 3 marzo 2016 Artículo aceptado: 13 julio 2016
}

\begin{abstract}
Resumen
Introducción: El pie diabético representa una de las causas de mayor morbilidad e incapacidad en las personas con Diabetes Mellitus. Esta población abarca hasta el 70\% de las amputaciones, en su mayoría desencadenada por la infección. La vasculopatía periférica, la neuropatía periférica asociadas al control metabólico juegan un rol importante en su génesis. El conocimiento de los mismos en la atención primaria asociadas a las medidas de prevención colaborarán en la disminución de este flagelo que aqueja a millones en la actualidad.

Objetivos: Determinar los factores de riesgo asociados a las lesiones del pie diabético. Describir las características demográficas y clínicas de los pacientes con lesiones del pie diabético.

Material y métodos: estudio de casos y controles. No probabilístico, de casos consecutivos. Se incluyeron pacientes adultos internados en el Departamento de Medicina Interna durante el 2015, diabéticos, con y sin lesiones en pie, sin amputaciones previas e independientes del tratamiento farmacológico.

Resultados: se incluyeron 86 sujetos, de los cuales 59\% de los casos presentaron onicomicosis, el 57\% neuropatía, el 67\% vasculopatía, 45\% algún tipo de deformidad y un 63\% utilizaban calzados inadecuados.

Conclusiones: La onicomicosis representó un factor de riesgo significativo para el desarrollo del pie diabético $(\mathrm{p}=0,02)$. En lo que respecta a la vasculopatía, la neuropatía y el control glicémico no se pudo establecer un riesgo significativo.
\end{abstract}

Palabras claves: pie diabético, polineuropatía diabética, angiopatía diabética, onicomicosis.

\begin{abstract}
Introduction: The diabetic foot is one of the main causes of higher morbidity and disability in people with Diabetes Mellitus. This population comprises up to 70\% of amputations, mostly triggered by infections. The peripheral vascular disease and the peripheral neuropathy associated with metabolic control play an important role in its genesis. The knowledge about them associated with prevention measures in primary care contribute to reduce this scourge that currently afflicts millions.
\end{abstract}

${ }^{1}$ Postgrado en Medicina Interna. Facultad de Medicina. Universidad Nacional de Itapúa (Encarnación, Paraguay)

Autor correspondiente:

Dr. Ángel Daniel Enciso Rojas.

Teléfono: +595.973555043

Correo electrónico: angelo_doc@hotmail.com 
Objectives: To determine risk factors associated with diabetic food lesions and to describe the demographic and clinical characteristics of patients with diabetic foot lesions.

Material and methods: Case-control study with non-probabilistic consecutive sampling. Adult diabetic patients with and without foot lesions, without previous amputations, independent of pharmacological treatment and admitted into the Department of Internal Medicine during 2015 were included.

Results: 86 subjects were included, 59\% of the cases had onychomycosis, 57\% neuropathy, 67\% vasculopathy, $45 \%$ some deformity and $63 \%$ used inadequate footwear. .

Conclusions: Onychomycosis represented a significant risk factor for the development of diabetic foot $(\mathrm{p}=$ 0.02). Concerning vasculopathy, neuropathy and glycemic control, a significant risk could not be established.

Keywords: diabetic foot, diabetic polyneuropathy, diabetic angiopathy, onychomycosis

\section{Introducción}

\section{Antecedentes}

La diabetes mellitus es uno de los principales problemas sanitarios y la demanda global de los sistemas de salud pública ha aumentado dramáticamente en las últimas dos décadas. Según los estudios epidemiológicos, el número de pacientes con diabetes mellitus ha aumentado de 30 millones de casos en 1985, a 177 millones en el año 2000, a 285 millones en el 2010 y se estima que si esta tendencia se mantiene, serán 360 millones las personas que padecerán esta enfermedad en los próximos 10 años ${ }^{1,2}$.

Las lesiones en el pie constituyen una de las mayores causas de morbilidad e incapacidad en las personas con diabetes. ${ }^{\text {(Jara) }}$ Representan la causa más frecuente de ingreso hospitalario en dicho grupo: se trata de una estancia hospitalaria prolongada y recurrente, y en ocasiones se precisa la amputación del miembro. Este hecho origina un coste social y económico elevado y una disminución de la calidad de vida de los pacientes ${ }^{3,4}$.

Entre 40\% y 70\% de las amputaciones de miembros inferiores ocurren en la población diabética, y hasta en $85 \%$ de los casos el factor desencadenante es la úlcera, asociada a la infección y gangrena ${ }^{5}$. La incidencia de un nuevo episodio tras una amputación ronda en torno al $50 \%$ a los 5 años ${ }^{3}$.

La lesión del pie diabético se define como toda infección, ulceración y/o destrucción de los tejidos profundos del pie asociados a trastornos neurológicos, vasculares y metabólicos (hiperglicemia sostenida) en extremidades inferiores de personas con diabetes ${ }^{6}$.

Partiendo de múltiples revisiones sistemáticas basadas experiencias clínicas, revisión de la literatura y estudios prospectivos se han podido establecer reglas de predicción clínica donde se describen varios factores de riesgo implicados en el desarrollo del pie diabético ${ }^{7,8}$. Los mismos son los siguientes: deformidad podálica, neuropatía periférica, enfermedad arterial periférica, niveles de HbA1c, presencia de callo plantar, tiempo de duración de la diabetes, tiña pedis y onicomicosis ${ }^{8,9}$.

La neuropatía periférica se define como la presencia de signos y síntomas de disfunción del sistema nervioso periférico en el paciente diabético, una vez excluida otras posibles causas ${ }^{10}$. Es una de las 
complicaciones microvasculares más importantes en la población diabética. Aumenta su prevalencia y gravedad conforme se incrementa el tiempo de evolución de la diabetes, la edad, la hiperglicemia y su duración. Constituye el factor de riesgo más importante asociado a la presencia de úlceras en el pie ${ }^{3}$. La literatura le atribuye entre un $50 \%$ a $60 \%$ de implicancia en el pie diabético ${ }^{2,11}$. Afecta a fibras nerviosas sensitivas, motoras y autonómicas del sistema nervioso periférico. La polineuropatía diabética más frecuente es la polineuropatía distal simétrica o sensitivomotora crónica; es asintomática en aproximadamente el 85\% de los casos. Puede iniciarse con la aparición de una úlcera en el pie si no se diagnostica en forma temprana y no se implementan medidas de prevención primaria ${ }^{3}$.

La polineuropatía sintomática engloba síntomas positivos que no se asocian a la presencia de signos objetivos positivos ni a un incremento en el riesgo de padecer lesiones en el pie. Entre ellos se encuentran las parestesias, disestesias, hipoalgesia, alodinia y el dolor quemante y/o urente. El dolor neuropático se caracteriza por empeorar a la noche y se localiza con más frecuencia en los pies y miembros inferiores, aunque en ocasiones afecta las manos. Los síntomas negativos como hipoalgesia, hipoestesia y anestesia se asocian a un aumento de riesgo de padecer lesiones ${ }^{3}$. Estas alteraciones propician la aparición de lesiones inducidas por una manipulación inadecuada de los pies, por el uso de calzados inapropiados y por hábitos higiénicos insuficientes. Esta iatrogenia pasa desapercibida y se mantiene en el tiempo debido a la pérdida del reflejo defensivo doloroso ${ }^{3}$.

Con respecto a las fibras motoras, ellas son las encargadas de mantener de forma correcta la posición de las articulaciones del pie, lo que permite una adecuada distribución de las presiones. Cuando éstas se alteran, se puede producir una atrofia muscular que induce a la deformidad ósea y modifica la biomecánica de la marcha y la redistribución de las presiones. La formación de callos en los puntos anómalos de presión, junto con un adelgazamiento de la cabeza metatarsiana, incrementa la presión plantar, y por último, induce al desarrollo de la úlcera ${ }^{3}$.

La pérdida de las fibras autonómicas produce una alteración en la regulación del flujo sanguíneo y una disminución de la sudoración; como consecuencia, la piel está seca y aparecen fisuras, puerta de entrada de infecciones ${ }^{3,12}$.

La enfermedad vascular periférica se caracteriza por la afectación distal al territorio infrapoplíteo, en ocasiones bilateral y multisegmentaria ${ }^{13}$. Si aparece la calcificación de la media se asocia a la presencia de neuropatía. Constituye un factor de riesgo determinante en la evolución de las lesiones del pie hacia la amputación ${ }^{3}$.

Es una manifestación del proceso aterosclerótico en el sistema arterial de los miembros inferiores que da lugar a una disminución del riego sanguíneo y en el peor de los casos, isquemia ${ }^{14}$. Se asocia a la presencia de afectación macrovascular en otras localizaciones y a un mayor riesgo de desarrollo de enfermedad cerebrovascular e infarto de miocardio (20\% a los 5 años $)^{3}$.

Entre los síntomas clásicos de la arteriopatía se describen la claudicación intermitente, como síntoma principal, además del dolor en reposo y en decúbito, la frialdad y la pérdida de vello ${ }^{15}$. A diferencia de la población sin diabetes, la arteriopatía en los diabéticos tiene una mayor afectación difusa y distal, que suele asociarse a la calcificación de la media en forma extensa. Habitualmente se acompaña de polineuropatía, por lo que la arteriopatía suele ser asintomática o se presenta con síntomas no clásicos ${ }^{16}$. El método diagnóstico más sensible (95\%) y específico (100\%) para la arteriopatía periférica en forma rápida e incruenta es la medición de la presión arterial en los 4 miembros y el cálculo del índice tobillo/brazo ${ }^{15}$. La presencia de afectación arterial periférica aumenta significativamente el riesgo de amputación, ya que no se puede satisfacer la demanda sanguínea necesaria para el control de una infección y una correcta cicatrización ${ }^{3,17}$. 
Las alteraciones biomecánicas están caracterizadas por modificar los puntos de apoyo durante la marcha o en posición estática, lo que induce a la aparición de callosidades y/o úlceras por presión. En casos extremos se puede originar la artropatía de Charcot, caracterizada por la aparición de fracturas y subluxaciones tras pequeños traumatismos, lo que ocasiona mayores deformidades, favoreciendo así la nueva producción de fracturas y de ulceraciones en los pies ${ }^{3,18,19}$.

La patología de la uña es la manifestación dermatológica más frecuente en el pie diabético, siendo la onicomicosis la afectación más común ${ }^{12}$. Aunque las onicomicosis no son más prevalentes en los diabéticos que en la población general, se caracterizan por ser una entidad de gran riesgo debido a sus posibles secuelas ${ }^{20}$. Habitualmente se observa engrosamiento ungueal, con una coloración blanco amarillenta típica y a menudo se presenta una ulceración subungueal secundaria a la presión sobre la uña distrófica. Puede existir un antecedente traumático, con pérdida de la uña y posterior crecimiento distrófico; va asociado a tiña pedis ${ }^{20,21}$.

La frecuencia en la aparición de lesiones del pie diabético es sumamente elevada; se estima que alrededor de un 15\% de las personas con diabetes presentarán algún compromiso a nivel del pie durante la evolución de la enfermedad ${ }^{22}$. Además la incidencia con respecto a las ulceraciones en pacientes con diabetes oscila el $2 \%{ }^{23}$.

El alto índice de recurrencia de estas lesiones y su alta tasa de mortalidad, convierten a esta patología en un importante problema de salud con elevada repercusiones sanitarias, económicas y sociales ${ }^{24}$.

Es de mencionar que los médicos generalistas y aún los especialistas se olvidan muchas veces de realizar una evaluación integral del paciente con la finalidad de llegar al diagnóstico precoz de la retinopatía, nefropatía y el pie diabético. Es por ello, que recae en la figura del Médico Internista llevar a cabo la identificación de los factores del riesgo asociados a las múltiples complicaciones de la diabetes mellitus ${ }^{18}$.

El propósito de esta investigación es indagar los factores de riesgo asociados a la aparición del pie diabético en pacientes que acuden al Hospital Nacional (Itauguá, Paraguay), centro de referencia para patologías complejas del la red de salud pública, de modo a guiar al Internista en la identificación y tratamiento precoz de las mismas a fin de prevenir la amputación del miembro con la carga médica y social que ello conlleva.

\section{Objetivos}

- Determinar los factores de riesgo asociados a las lesiones del pie diabético: presencia de pulsos arteriales en miembros inferiores, arteriopatía periférica, sensibilidad superficial en miembros inferiores, sensibilidad profunda en miembros inferiores, onicomicosis de pies, deformidad podálica, tipo de calzado, úlcera previa en pies, amputación previa de pies, tiempo de evolución de la diabetes, niveles HbA1c.

- Describir las características demográficas y clínicas de los pacientes con lesiones del pie diabético.

\section{Material y métodos}

Diseño: casos y controles.

\section{Población de estudio:}

Casos: varones y mujeres, mayores de edad, con lesiones del pie diabético de las salas de internación y consultorios del Dpto. de Medicina y Dpto. de Urgencias del Hospital Nacional (Itauguá, Paraguay) entre marzo y noviembre de 2015. 
Controles: varones y mujeres, mayores de edad, con diabetes mellitus y sin lesiones en los pies, de lassalas de internación y consultorios del Dpto. de Medicina y Dpto. de Urgencias del Hospital Nacional (Itauguá, Paraguay) entre marzo y noviembre de 2015.

\section{Criterios de inclusión:}

- Pacientes diabéticos tipo 1 o 2, independientemente del tratamiento farmacológico que reciban para el control de la enfermedad.

- Pacientes que han firmado el consentimiento informado.

\section{Criterios de exclusión:}

- Pacientes con amputaciones infracondíleasy/o supracondíleas previas.

- Pacientes con alteraciones del sensorio, afásicos o no colaboradores.

Muestreo: no probabilístico, de casos consecutivos.

\section{Variables:}

Demográficas: edad, sexo, procedencia, estado civil.

Clínicas:

- Predictoras: presencia de pulsos arteriales en miembros inferiores, arteriopatía periférica, sensibilidad superficial en miembros inferiores, sensibilidad profunda en miembros inferiores, onicomicosis de pies, deformidad podálica, tipo de calzado, úlcera previa en pies, amputación previa de pies, tiempo de evolución de la diabetes, niveles $\mathrm{HbA1}$.

- Resultante: úlceray/o necrosis del pie.

\section{Definiciones operacionales:}

Pulsos presentes: aquellas personas a las cuales al menos se pudo palpar el pulso pedio o tibial posterior, en ambos miembros inferiores. Se optó por la peor situación posible para considerar la existencia de enfermedad vascular periférica, es decir, que no se pudieran palpar los pulsos simultáneamente en al menos un miembro ${ }^{25}$.

Arteriopatía periférica: todo índice tobillo/brazo derecho e izquierdo inferior a 0,9 ${ }^{15,26,27}$.

Úlcera/amputación previa: se considera úlcera previa a toda lesión estadiable como como lesión estadío I, II, III, IV y V de la Clasificación de Wagner para pie diabético en uno o ambos pies de origen traumático o no. Como amputación previa se entiende toda resección de alguna sección anatómica de una o ambas extremidades inferiores según los niveles reseñados en el documento del Consenso internacional de pie diabético del $2007^{25}$.

Disminución de la sensibilidad superficial: se considera a aquellos pacientes que tras exploración con monofilamento no perciben el estímulo en 4 o más de los puntos preestablecidos en uno o ambos pies, en al menos, 2 ocasiones tras 3 intentos. La exploración se realiza con 10 puntos preestablecidos ( 9 en zona plantar y 2 en zona dorsal) que son: talón, arco interno, zona externa del pie, $1^{\mathrm{er}}, 2^{\mathrm{do}}, 3^{\mathrm{er}}$ metatarsiano, primer, tercer $\mathrm{y}$ quinto dedo, zona superior del $1^{\mathrm{er}} \mathrm{dedo}^{25,28}$.

Disminución de la sensibilidad profunda: se considera a aquellos pacientes que no perciben el estímulo tras la exploración de 3 puntos predeterminados (pulpejo del primer, primera cabeza del metatarsiano y maleolo interno) con diapasón en al menos 3 determinaciones, en uno o ambos pies ${ }^{25,28}$.

Deformidades del pie: se considera a las siguientes deformidades de la morfología podal normal ${ }^{19,25}$.

- Hallux adductus valgus: subluxación estática de la primera articulación metatarsofalángica con desviación lateral del primer dedo y desviación medial del primer metatarsiano con o sin pronación del dedo. 
- Pie plano: aplanamiento de los arcos longitudinales del pie que producen una huella plantar anormal.

- Pie cavo: aumento anormal de la altura de la bóveda plantar que produce una huella con falta de apoyo en la zona medial, con o sin acortamiento de pie.

- Dedos en garra: uno o varios dedos en donde existen una contractura en la flexión dorsal de la articulación metatarsofalángica, acompañada de la flexión dorsal de la articulación interfalángica proximal o de la interfalángica distal.

Calzado inadecuado: al que reúne uno de los siguientes parámetros ${ }^{25}$ :

- Calzado en mal estado (roto, desgastado o deformado por el uso).

- Calzado sin contrafuerte.

- Calzado que dejara expuesto los dedos o el talón.

- Calzado de punta estrecha que comprimiera los dedos.

- Calzado demasiado ancho que no permitiera un ajuste adecuado del pie.

- Calzado con un tacón de altura mayor a 2,5 cm.

- Calzado con costuras e imperfecciones en su interior que facilitan roces inadecuados.

Onicomicosis: infección micótica generalmente indolora, que se caracteriza por lo afectar a todas las uñas del pie ${ }^{20}$.

HbA1c: niveles mayores o iguales a 7,5 $\mathrm{mg} / \mathrm{dL}$ para confirmar diagnóstico de diabetes mellitus $\mathrm{y}$ monitoerear el tratamiento en los diabéticos conocidos ${ }^{11}$.

Instrumentos de medición: ficha técnica, tensiómetro electrónico, diapasón, estesiómetro

Cálculo de tamaño de muestra: se utilizó el programa estadístico EPI INFO $7^{\circledR}$. Se espera que los factores de riesgo más frecuentes sean la neuropatía periférica y la arteriopatía periférica.

Según referencias ${ }^{2,11}$, la neuropatía periférica se encuentra en $60 \%$ de los casos y $30 \%$ de los controles. Para un IC 95\%, razón 1:1, el tamaño mínimo calculado fue de 42 casos y 42 controles.

Según referencias ${ }^{11,29}$, la arteriopatía periférica se encuentra en $53 \%$ de los casos y $10 \%$ de los controles. Para un IC 95\%, razón 1:1, el tamaño mínimo calculado fue de 16 casos y 16 controles.

Finalmente, se incluyeron al menos 42 casos y 42 controles.

Reclutamiento: se acudió al libro de registro de ingresos para detectar a los pacientes internados en el Dpto. de Medicina Interna y el Dpto. de Urgencias. Los pacientes que acudían espontáneamente a las consultas en el horario de atención del autor fueron también incluidos, previo consentimiento informado.

Gestión de datos: las variables fueron registradas en fichas técnicas y trascriptas a planilla electrónica EXCEL. Luego fueron sometidas a estadística analítica con el programa EPI INFO $7^{\odot} 2000$. Las variables cualitativas se expresaron en porcentajes y las cuantitativas en medias \pm DS. Se calculó el OR con IC 95\%. Se consideró significativa toda $\mathrm{p}<0,05$. Para las variables cuantitativas se utilizó la prueba ANOVA o Kruskall Wallis, según distribución de las variables.

Control de calidad: sólo el autor llenó las fichas técnicas y realizó las maniobras semiológicas. Se realizó un pretest con 10 sujetos para detectar errores y corregirlos. Se utilizó tensiómetro electrónico para objetivar la presión arterial en los 4 miembros ${ }^{25}$. Los análisis laboratoriales se realizaron exclusivamente en el Dpto. de Laboratorio del Hospital Nacional. Los equipos de laboratorio fueron calibrados regularmente.

Aspectos éticos: se respetaron los Principios de la Bioética. Se mantuvo al máximo la confidencialidad de los datos personales. 
Justicia: todos los sujetos contactados tuvieron oportunidad de participar de la investigación. No se hizo discriminación por sexo, raza o creencia religiosa.

Beneficencia: los sujetos con lesiones arteriales o neuropáticas silentes fueron informados y remitidos a la consulta especializada. Los estudios fueron gratuitos.

Autonomía: los sujetos dieron su aprobación para la obtención de los datos previo consentimiento informado.

Este protocolo fue sometido a evaluación por el Comité de Ética de la Universidad Nacional de Itapúa. El autor declara que no existieron conflictos de interés comercial.

\section{Resultados}

En este estudio se incluyeron a 86 pacientes, de los cuales 43 correspondieron a los casos y 43 a los controles. La edad media fue de $56 \pm 14$ años (rango de $17-89$ años).

Hubo un ligero predominio del sexo masculino: 45 sujetos (52\%).

En lo que respecta a la procedencia de los sujetos en estudio, 43 (50\%) eran oriundos de distintos distritos del departamento Central y en segundo lugar, se sitúa Alto Paraná con 9 sujetos (10\%), luego le siguen Caaguazú y Paraguarí con 7 sujetos cada uno (8\%), Cordillera y Guairá con 5 (6\%) y 4 (5\%) respectivamente, San Pedro con 3 sujetos (3\%), Caazapá y Canindeyú con 2 sujetos cada uno (2\%) y Amambay, Concepción, Ñeembucú y Presidente Hayes con 1 cada uno (1\%).

El estado civil más frecuente fue la de casado: 66 sujetos (76\%).

Se pudo determinar que la mayoría de los sujetos en estudio eran portadores de la diabetes mellitus tipo 2: $82(95 \%)$.

Determinando los factores de riesgo asociados al pie diabético se encontró un riesgo significativo con la presencia de la onicomicosis (tabla 1).

Tabla 1

Factores de riesgo asociados al pie diabético.

\begin{tabular}{lcccc}
\hline \multicolumn{1}{c}{ Factor de riesgo } & Casos N: 43 & Controles N: 43 & OR (IC 95\%) & Valor p \\
\hline Neuropatía & $28(57 \%)$ & $21(43 \%)$ & $1,9(0,8-4,6)$ & 0,1 \\
Vasculopatía & $10(67 \%)$ & $5(33 \%)$ & $2,3(0,7-7,4)$ & $0,2^{*}$ \\
Onicomicosis & $33(59 \%)$ & $23(41 \%)$ & $2,8(1,1-7,2)$ & 0,02 \\
Deformidad & $23(59 \%)$ & $16(41 \%)$ & $1,9(0,8-4,5)$ & 0,1 \\
Calzado adecuado & $9(39 \%)$ & $14(61 \%)$ & $0,5(0,2-1,4)$ & 0,2 \\
\hline
\end{tabular}

* Corrección de Yates.

Las medias en relación al grado de control metabólico, los valores de ITB y el tiempo de evolución de la diabetes mellitus no resultaron estadísticamente significativas (tabla 2). 
Tabla 2

Factores de riesgo asociados al pie diabético.

\begin{tabular}{lccc}
\hline \multicolumn{1}{c}{ Factor de riesgo } & Casos N: 43 & Controles N: 43 & Valor $\mathbf{p}$ \\
\hline HbA1c & $10,1 \pm 2,5$ & $9,9 \pm 2,1$ & 0,6 \\
Índice tobillo-brazo & $1,03 \pm 0,1$ & $1,08 \pm 0,1$ & 0,1 \\
Años evolución DM & $11 \pm 8$ & $10 \pm 7$ & 0,5 \\
\hline
\end{tabular}

Sobre el total de sujetos en estudio, se pudo determinar que 39 (45\%) presentaron algún tipo de deformidad, de los cuales tanto el hallux adductus valgus 18 sujetos (46\%) como el pie plano 18 sujetos (46\%), fueron los principales hallazgos (tabla 3).

\section{Tabla 3}

Tipos de deformidad asociadas al pie diabético

\begin{tabular}{lcc}
\hline Tipo de deformidad & N & Porcentaje \\
\hline Dedos en garra & 3 & $8 \%$ \\
Hallux adductus valgus & 18 & $46 \%$ \\
Pie plano & 18 & $46 \%$ \\
Total & 39 & $100 \%$ \\
\hline
\end{tabular}

Se objetivó que el número de sujetos con calzado inadecuado fue 63 (73\%). El tipo de calzado que predominó fue sin contrafuerte: 47 (75\%) (tabla 4).

Tabla 4

Tipos de calzado.

\begin{tabular}{lcc}
\hline \multicolumn{1}{c}{ Tipo de Calzado } & N & Porcentaje \\
\hline Comprime los dedos & 14 & $22 \%$ \\
Punta estrecha & 2 & $3 \%$ \\
Sin contrafuerte & 47 & $75 \%$ \\
Total & 63 & $100 \%$ \\
\hline
\end{tabular}

\section{Discusión}

Los pacientes diabéticos tienen un elevado riesgo de padecer complicaciones crónicas cardiovasculares, renales, oftalmológicas, neurológicas y podológicas cuyas incidencias crecen anualmente. Dentro de estas, el síndrome de pie diabético tiene implicaciones muy importantes en morbilidad, discapacidad y deterioro de la 
calidad de vida de los pacientes diabéticos, además de hacer muchas veces más difícil su acceso a los servicios de salud por las limitaciones físicas que impone. La úlcera del pie diabético puede ocurrir en más del 15\% de los pacientes diabéticos durante su vida y aproximadamente 14-24\% de los pacientes con úlcera del pie diabético requerirán una amputación ${ }^{18}$.

La neuropatía diabética y la enfermedad arterial periférica contribuyen al incremento de la morbilidad y la mortalidad por pie diabético, hecho que genera un gran impacto económico en el sistema de salud, y que, por tanto, constituye un problema de salud pública que influye en la calidad de vida de las personas afectadas y sus familias ${ }^{18}$.

La identificación de la neuropatía diabética y de la enfermedad arterial periférica por medio de la anamnesis y el examen físico y las recomendaciones de cuidado, son actividades preventivas subutilizadas, a pesar de ser consideradas como intervención de primera línea, dado que representan una estrategia costoefectiva en la disminución del riesgo de desarrollo de las complicaciones del pie diabético ${ }^{18}$.

La utilización del monofilamento Semmes-Weinstein es una herramienta sencilla, práctica y precisa utilizada como prueba de detección de neuropatía diabética, ya que proporciona una medición de manera estandarizada de la sensibilidad que percibe el paciente al realizar presión en puntos específicos ${ }^{30}$.

Inicialmente se utilizó como un indicador pronóstico específico de la presencia de infecciones en piel, úlceras y amputación. Actualmente la prueba de monofilamento es una de las más utilizadas para identificar neuropatía diabética y tiene una sensibilidad del 78\% y una especificidad del 96\% para identificar la presencia de neuropatía diabética ${ }^{30}$.

En este estudio, la neuropatía estuvo presente en el $57 \%$ de los casos y en el $43 \%$ de los controles; se pudo demostrar que la misma puede ser catalogada como un factor de riesgo pero su asociación con la presencia del pie diabético no fue significativa: OR 1,9 (IC 95\% 0,8-4,6) (p 0,1). Los hallazgos distan de los resultados obtenidos en estudios de similares características metodológicas como el trabajo del Centro Cardiovascular de Barranquilla: IC $(\mathrm{OR} 4,95)(\mathrm{p}<0,05)$, donde sí se pudo establecer una asociación significativa ${ }^{8}$. Posiblemente el tamaño de muestra incluido en este estudio no haya sido suficiente para demostrar esta relación.

En lo que respecta a la vasculopatía periférica, se la pudo objetivar en el $67 \%$ de los casos y en el 33\% de los controles. En este estudio, en similitud con los hallazgos de la neuropatía, los resultados pudieron establecer a esta entidad como un factor de riesgo para el desarrollo del pie diabético pero no fue estadísticamente significativa: OR 2,3 (IC 95\% 0,7-7,4) (p 0,2). Tomando como referencia el estudio del Centro Cardiovascular de Barranquilla, donde se pudo establecer una fuerte relación con significancia estadística: OR 13,62 (IC 6,23$30,34)(p<0,001)^{8}$, no se pudo demostrar en este estudio. Tal vez aumentando el tamaño de muestra se detecte esta relación entre variables.

Algo que resulta llamativo en este estudio fue la fuerte asociación que se estableció entre la presencia de onicomicosis y la posibilidad de desencadenar lesiones compatibles con el pie diabético: OR 2,8 (IC 95\% 1,1$7,2)(p 0,02)$. En toda la literatura sometida a revisión sistemática, no se encontraron estudios que hayan podido establecer una significancia estadística como lo fue en esta experiencia ${ }^{31}$.En este trabajo, la onicomicosis estuvo presente en el 59\% de los casos y en el 41\% de los controles. Es sabido que las lesiones micóticas del pie son puerta de entrada de infecciones que pueden llevar a gangrenas ${ }^{32}$.

Con respecto a la media de la HbA1c en los sujetos que formaron parte de este estudio, se identificó en los casos una media de 10,1 $\pm 2,5 \%$ y en los controles $9,9 \pm 2,1 \%$. En un metaanálisis ${ }^{34}$ se pudo establecer una 
relación significativa entre los niveles de la HbA1c y la amputación del pie diabético, la cual no pudo ser verificada en esta experiencia. Lo que podría deberse a la diferencia en el tamaño de muestra de un estudio con respecto al otro.

La media del ITB (Índice tobillo brazo) en los casos fue 1,03 \pm 0,1 y en los controles 1,08 \pm 0,1 con un valor de la p 0,1. Como queda claro, no se determinó que exista una relación estrecha entre los hallazgos de esta medición y la posibilidad de desarrollar pie diabético en el futuro. Esto podría explicarse a la necesidad de contar con métodos auxiliares que coadyuven la medición ITB y aumenten su sensibilidad y especificidad tales como el análisis simultáneo de la morfología de la onda del pulso, normalmente trifásica ${ }^{3}$. Recientes estudios de medición de ITB en sujetos jóvenes sanos han sugerido que los valores normales tradicionales de este índice deberían aumentar ${ }^{35}$.

El tiempo de duración de la diabetes en los casos presentó una media de $11 \pm 8$ años y en los controles de 10 \pm 7 años (p 0,5). Es decir, no se corroboró una asociación significativa entre el tiempo de la evolución de la enfermedad y la aparición del pie diabético. Clásicamente se ha considerado que padecer la enfermedad por más de 10 años de evolución favorecía la progresión hacia el desarrollo del pie diabético ${ }^{24}$. Este paradigma ha sido sistemáticamente rechazado en estudios como la del Centro Cardiovascular de Barranquilla donde no se corroboró esta asociación: $\mathrm{OR}=0,96^{8}$. Pero ha sido fuertemente establecido en estudios como los realizados en Hospitales de Tijuana, México ${ }^{36}$.

Las deformidades podálicas fueron descritas durante este estudio como factores de riesgo para la progresión hacia el pie diabético. Dentro de las distintas deformidades descritas pudimos establecer que 39 (45\%) de los sujetos presentan algún tipo de deformidad, siendo el hallux adductus valgus (48\%) y el pie plano (48\%) las más frecuentes. Comparado el estudio mexicano se pudo encontrar resultados similares ${ }^{36}$. En este estudio se pudo establecer que la deformidad podálica representaba un riesgo para la presencia del pie diabético: OR 1,9 (IC 95\% 0,8-4,5) pero la misma no fue estadísticamente significativa. Algún tipo de deformidad estuvo presente en el $59 \%$ de los casos y en el $41 \%$ de los controles. Tal vez aumentando el tamaño de muestra se logre objetivar la relación entre estas variables.

En lo que respecta al tipo de calzado, tomando como parámetro si era adecuado o no, se determinó el uso de calzado adecuado en 39\% de los casos y en 61\% de los controles. Con un 0R 0,5 (IC 95\% 0,2-1,4) se concluyó que el uso de calzado adecuado en pacientes diabéticos implicaba un efecto protector para la aparición del pie diabético pero esta afirmación desde el punto de vista estadístico no fue significativo (p 0,1). De los sujetos en estudio, el 73\% (63 sujetos) empleaban un calzado inadecuado. De las distintas variables asignadas como calzado inadecuado, un 75\% correspondía a los de tipo sin contrafuerte. Se prescribe calzado protector a los pacientes de alto riesgo de presentar neuropatía y deformidades; la intención es reducir la presión plantar elevada en ciertas áreas y la mayor fricción con redistribución de la presión en una mayor área de superficie ${ }^{37}$.

Las medidas del zapato (amplitud y profundidad) se diseñan de acuerdo con las deformidades, al igual que las plantillas y demás ortesis; lo ideal es el calzado protector con cordones o velcro para brindar ajuste al pie de acuerdo con el grosor de las medias o la presencia de edema según la hora, sin costuras internas, tacón de 2-3cm para evitar sobrecarga en el antepié, con puntera ancha y alta que permita la movilidad de los dedos; además, con plantillas moldeadas. Este tipo de calzado se debe usar permanentemente, tanto en casa como fuera de ésta y es necesario advertirle al paciente que no camine descalzo ${ }^{37}$.

En la gran mayoría de los pacientes con Diabetes Mellitus, el desarrollo de una úlcera en pies se pudo prevenir. La educación a los pacientes sobre higiene de los pies, cuidados de la piel, cuidado de las unas, uso de calzado apropiado y la atención adecuada de los pies por profesionales calificados puede reducir las 
lesiones que puedan conducir a la ulceración del pie ${ }^{36}$.

Lastimosamente, en esta investigación no se evaluó la educación de los pacientes con respecto a las medidas de prevención ${ }^{38}$. Se debería realizar una investigación más exhaustiva de las condiciones socioeconómicas de nuestra población; a partir de la cual se puedan tomar medidas establecidas en los protocolos de prevención primaria y secundaria, generando de esta manera un impacto favorable en nuestros pacientes y contribuyendo a retrasar la aparición del pie diabético, según las recomendaciones internacionales ${ }^{39}$.

Es costo efectivo implementar los programas y las actividades de prevención para retrasar las complicaciones. Por lo anterior, los médicos generales y especialistas tienen la responsabilidad de hacer prevención en todos los niveles de atención, para frenar el desarrollo de la diabetes mellitus y de las lesiones crónicas secundarias. Asimismo, es prioritario educar al paciente y su familia para que realicen el autocuidado y el automonitoreo del tratamiento ${ }^{18}$.

En conclusión, la mayoría de los pacientes involucrados en este estudio eran oriundos de localidades del Departamento Central, predominantemente del sexo masculino y con una edad promedio de 56 años.

Hubo una gran prevalencia de pacientes casados y la diabetes mellitus tipo 2 fue la más frecuente entre los pacientes en estudio.

Tanto la neuropatía como la vasculopatía periférica demostraron ser factores de riesgo para la presencia del pie diabético pero no resultaron estadísticamente significativas. Este mismo hecho se planteó tanto para la presencia de deformidades podálicas como para el tipo de calzado.

Dentro de las variables estudiadas como factores de riesgo asociados a la aparición del pie diabético, la presencia de onicomicosis se destacó por haber sido estadísticamente significativa.

La medida de control metabólico (HbA1c) de los sujetos en estudio fue de 10\%, el tiempo de evolución de la enfermedad rondó los 10 años y las mediciones de ITB promediaron 1,08.

Las deformidades podálicas más frecuentes fueron el hallux adductus valgus y el pie plano. La gran mayoría de nuestros pacientes no contaban con calzados adecuados.

\section{Referencias bibliográficas}

1. Janmohammadi N, Hasanjani Roushan MR, Moazezi Z, Rouhi M, Esmailnejad Gangi SM, Bahrami M. Epidemiological characteristics of diabetic foot ulcer in Babol, north of Iran: a study on 450 cases. Caspian J Intern Med. 2011l; 2(4):321-5.

2. Yazdanpanah L, Nasiri M, Adarvishi S. Literature review on the management of diabetic foot ulcer. World J Diabetes. 2015; 6(1):37-53.

3. Gómez Hoyos E, Levy AE, Díaz Pérez A, Cuesta Hernández M, Montañez Zorrilla C, Calle Pascual AL. Pie diabético. Semin Fund Esp Reumatol. 2012; 13(4):119-29.

4. Cisneros Lde L, Fonseca TH, Abreu VC. Inter- and intra-examiner reliability of footprint pattern analysis obtained from diabetics using the Harris mat. Rev Bras Fisioter. 2010; 14(3):200-5. 
5. Llanes Barrios JA, Álvarez Duarte HT, Toledo Fernández AM, Fernández Montequín JI, Torres Herrera OF, Chirino Carreño N, et al. Manual para la prevención, diagnóstico y tratamiento del pie diabético. Rev Cubana Angiol Cir Vasc [Internet]. 2009 [citado 10 Nov. 2010]; 10(1): 42-96. Disponible en: https://es.scribd.com/doc/220279748/Manual-Para-La-Prevencion-Diagnostico-y-Tratamiento-DelPie-Diabetico

6. Seguel G. Por qué debemos preocuparnos del pie diabético? Importancia del pie diabético. Rev Méd Chile. 2013; 141(11):1464-9.

7. Chia-Mou L, Chang-Cheng Ch, Mei-Yu P, Chyong-Fang Ch, Mei-Yen Ch. Insufficient early detection of peripheral neurovasculopathy and associated factors in rural diabetes residents of Taiwan: a crosssectional study. BMC Endocr Disord. 2014; 14:89.

8. Rosalis Amaris M, Bonilla Rojas J, Gómez Batista A, Gómez Chaparro C, Pardo García J, Villanueva Rodríguez L. Factores asociados al pie diabético en pacientes ambulatorios. Centro de Diabetes Cardiovascular del Caribe. Barranquilla (Colombia). Salud Uninorte. Barranquilla (Col.). 2012; 28(1):65-74.

9. Crawford F, Anandan C, Chappell FM, Murray GD, Price JF, Sheikh A, et al. Protocol for a systematic review and individual patient data meta-analysis of prognostic factors of foot ulceration in people with diabetes: the research collaboration for the prediction of diabetic foot ulcerations (PODUS). BMC Med Res Methodol. 2013; 13:22.

10. Fawzy OA, Arafa AI, El Wakeel MA, Abdul Kareem SH. Plantar pressure as a risk assessment tool for diabetic foot ulceration in egyptian patients with diabetes. Clin Med Insights Endocrinol Diabetes. 2014; 7:31-9.

11. Porciúncula MVP, Rolim CCP, Garofolo L, Ferreira SRG. Análise de fatores associados à ulceração de extremidades em indivíduos diabéticos com neuropatia periférica. Arq Bras Endocrinol Metabol. 2007; 51(7):1134-42.

12. Martos Medina D, Roldán Valenzuela A. Infecciones dermatológicas en pacientes diabéticos. /Internet/. /citado 10 Nov. 2010/. Disponible en: http://www.podologo.cl/podologo/documentos/ INFECCIONESDIABETICOS.pdf

13. García Viejo A. Enfermedad arterial periférica y pié diabético en pacientes en programa de hemodiálisis. Enferm Nefrol. 2012;15(4):255-64.

14. Lepäntalo M, Fiengo L, Biancari F. Peripheral arterial disease in diabetic patients with renal insufficiency: a review. Diabetes Metab Res Rev. 2012; 28 Suppl 1:40-5.

15. Serrano Hernando FJ, Martín Conejero A. Peripheral artery disease: pathophysiology, diagnosis and treatment. Rev Esp Cardiol. 2007; 60(9):969-82.

16. Rodríguez Gurri D, González Expósito A. Caracterización de pacientes con pie diabético. Rev Cub Med Mil. 2013; 42(2):173-80.

17. Peters EJ, Lipsky BA, Berendt AR, Embil JM, Lavery LA, Senneville E, et al. A systematic review of the effectiveness of interventions in the management of infection in the diabetic foot. Diabetes Metab Res Rev. 2012;28 Suppl 1:142-62.

18. Pinilla AE, Barrera MP, Rubio C, Devia D. Actividades de prevención y factores de riesgo en diabetes mellitus y pie diabético. Acta Med Colomb. 2014;39(3):250-57.

19. Varma AK. Charcot neuroarthropathy of the foot and ankle: a review. J Foot Ankle Surg. 2013; 52(6):740-9.

20. Giménez AM, Riambau V, Escudero JR. Lesiones cutáneas asociadas al pie diabético. /Internet/. /citado 10 Nov. 2010/.Disponible en: http://www.sld.cu/galerias/pdf/sitios/rehabilitacion/lesiones_cutaneas_ asociadas_al_pie_diabetico.pdf

21. Rodríguez Gurri D. Implicación del traumatismo en la fisiopatología del pie diabético. Rev Cub Med Mil. 2014; 43(3):370-8.

22. Rivero Fernández F, Expósito Martín T, Rodríguez Alonso ME, Lazo Díaz I. Frecuencia de amputaciones por pie diabético en un área de salud. Arch Méd Camaguey /Internet/ 2005 /citado 10 Nov. 2010/; 9(2). Disponible en: http://www.amc.sld.cu/amc/2005/v9n2/1048.htm

23. Martínez Sabater A, Pascual Ruíz MF. Valoración del riesgo de pie diabético en el paciente anciano en una consulta de enfermería. Gerokomos. 2009;20(2):73-7.

24. Santos IC, Sobreira CMM, Nunes EM, Morais MC. Prevalência e fatores associados a amputações por pé diabético. Ciênc saúde coletiva. 2013;18(10):3007-14. 
25. González de la Torre H, Perdomo Pérez E, Quintana Lorenzo M, Mosquera Fernández A. Estratificación de riesgo en pie diabético. Gerokomos. 2010;21(4):172-82.

26. Martín Borge V, Herranz de la Morena L, Castro Dufourny I, Fernández Martínez A, Pallardo Sánchez LF. Factores de riesgo y pie diabético. An Med Interna. 2007 Jun; 24(6):263-6.

27. Vega J, Romaní S, Garcipérez FJ, Vicente L, Pacheco N, Zamorano J, Gómez-Barrado JJ, Sánchez MuñozTorrero JF. Peripheral arterial disease: efficacy of the oscillometric method. Rev Esp Cardiol. 2011l; 64(7):619-21.

28. Skopljak A, Sukalo A, Batic-Mujanovic 0, Muftic M, Tiric-Campara M, Zunic L. Assessment of diabetic polyneuropathy and plantar pressure in patients with diabetes mellitus in prevention of diabetic foot. Med Arch. 2014; 68(6):389-93.

29. Martin IS, Beraldo AA, Passeri SM, Freitas MCF, Pace AE. Causas referidas para o desenvolvimento de úlceras em pés de pessoas com diabetes mellitus. Acta paul enferm. 2012; 25(2):218-24.

30. Perkins BA, Olaleye D, Zinman B, Bril V. Simple screening tests for peripheral neuropathy in the diabetes clinic. Diabetes Care. 2001; 24(2):250-6.

31. Larruskain Garmendia J, Idígoras Viedma P, Mendiola Arza J. Onicomicosis: diagnóstico y tratamiento. Inf Ter Sist Nac Salud. 2008; 32(3):83-92.

32. Volmer-Thole M, Lobmann R. Neuropathy and diabetic foot syndrome. Int J Mol Sci. 2016; 17(6). pii: E917.

33. Malgrange D. Physiopathology of the diabetic foot. Rev Med Interne. 2008; 29 Suppl 2: S231-7.

34. Papanas N, Maltezos E. Glycated hemoglobin as a risk factor for lower extremity amputations in diabetes: success is counted sweetest. Int J Low Extrem Wounds. 2015; 14(2):106-7.

35. Quong WL, Fung AT, Yu RY, Hsiang YN. Reassessing the normal toe-brachial index in young healthy adults. J Vasc Surg. 2016; 63(3):652-6.

36. Márquez-Godínez SA, Zonana-Nacach A, Anzaldo-Campos MC, Muñoz-Martínez JA. Riesgo de pie diabético en pacientes con diabetes mellitus tipo 2 en una unidad de medicina de familia. Semergen. 2014; 40(4):183-8.

37. Apelqvist J, Bakker K, van Houtum WH, Schaper NC. Practical guidelines on the management and prevention of the diabetic foot: based upon the International Consensus on the Diabetic Foot (2007) Prepared by the International Working Group on the Diabetic Foot. Diabetes Metab Res Rev. 2008 May-Jun; 24 Suppl 1:S181-7.

38. Kishore S, Upadhyay AD, V P J. Awareness of foot care among patients with diabetes attending a tertiary care hospital. Natl Med J India. 2015 May-Jun; 28(3):122-5.

39. Hingorani A, LaMuraglia GM, Henke P, Meissner MH, Loretz L, Zinszer KM, et al. The management of diabetic foot: a clinical practice guideline by the Society for Vascular Surgery in collaboration with the American Podiatric Medical Association and the Society for Vascular Medicine. J Vasc Surg. 2016 Feb; 63(2 Suppl): 3S-21S. 